\title{
Keratoderma hereditarium mutilans
}

INSERM

\section{Source}

INSERM. (1999). Orphanet: an online rare disease and orphan drug data base.

Keratoderma hereditarium mutilans. ORPHA:494

Keratoderma hereditarium mutilans is a rare, diffuse, mutilating, hereditary palmoplantar keratoderma disorder characterized by severe, honeycomb-pattern palmoplantar keratosis and pseudoainhum of the digits leading to autoamputation, associated with mild to moderate congenital sensorineural hearing loss. Additional features include stellate keratosis on the extensor surfaces of the fingers, feet, elbows and knees. Alopecia, onychogryphosis, nail dystrophy or clubbing, spastic paraplegia and myopathy may also be associated. 